HWR No. 04-02

\title{
Estimation of Effective Unsaturated Hydraulic Conductivity Tensor Using Spatial Moments of Observed Moisture Plume
}

\author{
by \\ Tian-Chyi J. Yeh, Ming Ye \\ and \\ Raziuddin Khaleel
}

Department of Hydrology and Water Resources

University of Arizona

Tucson Arizona 85721 


\title{
Estimation of Effective Unsaturated Hydraulic Conductivity Tensor Using Spatial Moments of Observed Moisture Plume
}

\author{
Tian-Chyi J. Yeh ${ }^{1^{*}}$, Ming Ye ${ }^{2}$, and Raziuddin Khaleel ${ }^{3}$
}

October 2004

For Publication in Water Resources Research

${ }^{1}$ Department of Hydrology and Water Resources, The University of Arizona, Tucson, AZ 85721

${ }^{2}$ Desert Research Institute, University and Community College System of Nevada, 755 E. Flamingo Road, Las Vegas, NV 89119, USA

${ }^{3}$ Fluor Government Group, P. O. Box 1050, Richland, WA 99352

*Corresponding Author 
1

2

3

4

5

6

7

8

9

\begin{abstract}
Knowledge of unsaturated zone hydraulic properties is critical for many environmental and engineering applications. Various stochastic methods have been developed during the past two decades to estimate the effective unsaturated hydraulic properties. Independent of these stochastic methods, we develop in this paper a practical approach to estimate the threedimensional (3-D) effective unsaturated hydraulic conductivity tensor using spatial moments of 3-D snapshots of a moisture plume under transient flow conditions. Application of the new approach to a field site in southeastern Washington State yields an effective unsaturated hydraulic conductivity tensor that exhibits moisture-dependent anisotropy. The effective hydraulic conductivities compare well with laboratory-measured unsaturated hydraulic conductivity data from small core samples; they also reproduce the general behavior of the observed moisture plume at the site. We also define a moisture diffusivity length concept which we use in conjunction with estimated correlation scales of the geological media at the field site to explain deviations between the observed and simulated plumes based on the derived effective hydraulic properties.
\end{abstract}


1

\section{INTRODUCTION}

Geologic formations are heterogeneous at various length scales. To describe the bulk (or mean) flow behavior of a heterogeneous formation, the formation is often replaced by its homogeneous equivalent and effective or upscaled flow parameters are used to represent the equivalent medium. This study is concerned with deriving the bulk behavior of large scale unsaturated media. The large scale corresponds to a scale affected by releases from a point injection source - a length scale of tens of meters in the lateral as well as in the vertical direction.

Numerous studies have investigated the effective flow and transport properties for geological formations under saturated conditions (Gelhar, 1993) but very few studies have examined the effective properties for unsaturated media. Theoretical work (e.g., Yeh et al., 1985a, b and c; Mantoglou and Gelhar, 1987; Green and Freyberg, 1995), numerical simulations (e.g., Khaleel et al., 2002; Wildenschild and Jensen, 1999b; Desbarats, 1998; Lu and Khaleel, 1993; Polmann et al., 1991; Yeh, 1989; Ababou 1988) and experimental studies (e.g., Wildenschild and Jensen, 1999a; McCord et al., 1991; Yeh and Harvey, 1990; Stephens and Heerman, 1988) suggest that the effective unsaturated hydraulic conductivity $(K)$ tensor for stratified sediments can exhibit moisture or tension dependent anisotropy. That is, the anisotropy (ratio of $K$ parallel to bedding to $K$ perpendicular to bedding) increases with increasing tension (or decreasing moisture content, $\theta$ ). While the moisture dependent anisotropy has often been invoked in modeling flow in large-scale unsaturated media, the concept has not been widely tested and verified in the field. This can be attributed to the fact that, although a number of large-scale field experiments (e.g., Rudolph et al., 1996; McCord et al., 1991; Wierenga et al., 1991a, b) have been conducted in the past, very few of these experiments have collected sufficiently dense pressure head or moisture content data to quantify its presence in the field. 
1 Recently, Gee and Ward (2001) conducted water and tracer injection experiments at a field site

2 located within the U. S. Department of Energy Hanford Site in southeastern Washington. Using

3 neutron probes, the initial and subsequent $\theta$ fields were obtained for 32 wells and 43 depths

4 during the injection experiment. Such an extensive and dense data set provides a unique

5 opportunity to investigate effects of heterogeneity on the dynamics of moisture movement within

6 the vadose zone and evaluate moisture dependent anisotropy in the effective $K$ at the field site.

7 Generally, field applications of the stochastic models to describe effective unsaturated

8 hydraulic properties (Yeh et al., 1985 a, b, and c, Mantoglou and Gelhar, 1987, and Polmann et

9 al.,1991) require accurate spatial statistics of unsaturated media properties at small scales (Holt

10 et al., 2002). This requirement, in turn, demands extensive sampling of field sites and hydraulic

11 properties measurements, which are difficult, time consuming and costly (e.g., Greenholtz et al.,

12 1988). Therefore, alternative approaches for estimating the effective unsaturated hydraulic

13 properties and associated moisture-dependent anisotropy in situ are needed.

14 In this paper, we develop a practical approach, which uses snapshots of observed

15 moisture content plume under transient flow conditions to derive a three-dimensional (3-D)

16 effective $K$ tensor. The methodology uses the temporal evolution of spatial moments of

17 observed $\theta$ plumes and is independent of the preceding stochastic theories describing effective

18 unsaturated hydraulic properties. Our new approach is similar to those of Sudicky (1986),

19 Freyberg (1986) and Barry and Sposito (1990) who tied the moment analysis of the Borden

20 tracer data to the macrodispersion concept in saturated media. The new technique is applied to

21 the field site (Gee and Ward, 2001) and the results are compared with laboratory-measured

22 unsaturated media properties (Khaleel and Freeman,1995; Khaleel et al.,1995) at the site. 
1 Subsequently, using the derived effective hydraulic properties, we simulate the evolution of the

2 3-D moisture plume due to the injection experiment. The results are then compared with the

3 observed moisture plume. Finally, we define a variable moisture diffusivity length concept and

4 use its relation to the spatial correlation scale of the geologic media at the field site to evaluate

5 the effectiveness of our effective hydraulic conductivity parameter estimation approach.

\section{2. APPROACH}

As stated earlier, stochastic theories relate the effective unsaturated hydraulic properties

9 of an equivalent homogeneous medium to the spatial statistics (i.e., means, variances, and 10 correlation scales) of properties measured at small scales. As described below, instead of relying on the spatial statistics of small-scale hydraulic properties, the new method directly derives the effective properties using observed responses, specifically, the spatial and temporal changes in $\theta$ within the vadose zone. This is similar to the approach used by Yeh (1989), who used an

\subsection{Spatial Moments of the Moisture Plume.}

Spatial moments (Aris, 1956) have been widely used for quantifying migration and spread of a solute plume (e.g., Sudicky, 1986). The spatial moment approach has recently been used to quantify the migration and spread of a moisture plume (e.g., Gee and Ward, 2001; Ye et al. 2004; this issue). In particular, the spatial moments for a moisture plume are defined as

$$
M_{i j k}(t)=\int_{-\infty}^{+\infty} \int_{-\infty}^{+\infty} \int_{-\infty}^{+\infty} \theta_{d i f f}(x, y, z, t) x^{i} y^{j} z^{k} d x d y d z
$$


1 where $\theta_{\text {diff }}(x, y, z, t)=\theta(x, y, z, t)-\theta_{\text {init }}(x, y, z, 0) ; \theta(x, y, z, t)$ represents the moisture content at a

2 given time $t$ at a location $x, y, z$, and $\theta_{\text {init }}(x, y, z, 0)$ represents the initial moisture content. The

3 zeroth, first and second spatial moments correspond to $i+j+k=0,1$, and 2 , respectively. The

4 zeroth moment $\left(M_{000}\right)$ represents the changes in moisture storage within the domain. The

5 location $\left(x_{c}, y_{c}, z_{c}\right)$ of mass center of the moisture content difference at a given time is

6 represented by

$$
x_{c}=M_{100} / M_{000}, y_{c}=M_{010} / M_{000}, \text { and } z_{c}=M_{001} / M_{000} \text {. }
$$

8 The spread of the plume about its center is described by the spatial variance in $x, y$, and $z$ 9 directions:

$$
\sigma_{x x}=\frac{M_{200}}{M_{000}}-x_{c}^{2} \quad \sigma_{y y}=\frac{M_{020}}{M_{000}}-y_{c}^{2} \quad \sigma_{z z}=\frac{M_{002}}{M_{000}}-z_{c}^{2} .
$$

\subsection{Estimation of Effective Unsaturated Hydraulic Properties.}

We now relate the calculated spatial moments to the effective unsaturated hydraulic properties. Consider a vadose zone that can be represented by an equivalent homogeneous medium. A $\theta$-based Richards' equation can be used to describe the moisture movement in the medium under unsaturated conditions:

$$
\nabla \cdot(D(\theta) \nabla \theta)+\frac{\partial K_{z}(\theta)}{\partial \theta} \frac{\partial \theta}{\partial z}=\frac{\partial \theta}{\partial t}
$$

where $D(\theta)$ is the effective moisture diffusivity tensor, and $K_{z}(\theta)$ is the effective unsaturated hydraulic conductivity in the vertical $(z)$ direction. Note that a $\theta$-based Richards' equation is valid only for the partially saturated homogeneous media, where $D(\theta)$ and $K(\theta)$ relations are spatially invariant over the entire medium. Although the vadose zone is inherently heterogeneous at small observation scales (i.e., core scale), it can be visualized as an equivalent 
1 homogeneous medium either in an ensemble mean or in a spatial average sense. If a REV

2 exists, $\theta$ in equation (4) represents the average of moisture content over many locations at an

3 observation scale whose length scale is much greater than the heterogeneity scale (i.e., a spatially

4 averaged value). To the contrary, if a REV does not exist, then it denotes the $\theta$ in a fictitious

5 medium, representing the moisture content averaged over many possible vadose zones (i.e.,

6 ensemble mean $\theta$ ). The REV is defined as a control volume or control volumes whose volume-

7 averaged properties are representative of every part of the medium in the field regardless of the

8 location of the control volume in the medium (de Marsily, 1986). Despite possible differences

9 between the observed point values of $\theta$ and those by equation (4), the aim of the equivalent

10 homogeneous approach is to produce the best, unbiased estimates of spatial moments of $\theta$

11 distribution within the vadose zone.

12 Notice that equation (4) is analogous to an advection-dispersion equation for solute

13 transport, where the first term on the left-hand side represents dispersion (i.e., the influence of

14 capillary forces in dispersing a moisture front) and the second term represents the convective

15 term (i.e., the effect of gravity causing downward propagation of moisture at a rate determined

16 by $\left.\partial K_{z} / \partial \theta\right)$. Accordingly, the convective term represents the rate of change of the first spatial

17 moment of $\theta$ in the $z$ direction, i.e.,

$$
\frac{\partial K_{z}}{\partial \theta}=V_{z}(\theta)=\left.\frac{d z_{c}}{d t}\right|_{t=f(\theta)}
$$

19 The vertical hydraulic conductivity, $K_{z}$, consequently can be estimated provided that information on vertical velocity, $\mathrm{V}_{\mathrm{z}}$ is available for different $\theta$ values.

Following the molecular diffusion or solute dispersion analysis that relates the rate of change in the spatial variance of a tracer plume to its diffusion or dispersion coefficient 
1 (Csanady,1973; Fisher et al., 1979), the rate of change in the spatial variance for a given $\theta$ can be

2 related to moisture diffusivity:

3

4

6 the anisotropy in $K$, i.e.,

$7 \frac{K_{x}(\theta)}{K_{z}(\theta)}=\frac{D_{x}(\theta)}{D_{z}(\theta)}=\frac{\partial \sigma_{x x}^{2}(\theta) / \partial t}{\partial \sigma_{z z}^{2}(\theta) / \partial t}$ and $\frac{K_{y}(\theta)}{K_{z}(\theta)}=$
8 Furthermore, $K_{x}(\theta)$ and $K_{y}(\theta)$ functions can be derived using

$$
\frac{K_{x}(\theta)}{K_{z}(\theta)}=\frac{D_{x}(\theta)}{D_{z}(\theta)}=\frac{\partial \sigma_{x x}^{2}(\theta) / \partial t}{\partial \sigma_{z z}^{2}(\theta) / \partial t} \quad \text { and } \quad \frac{K_{y}(\theta)}{K_{z}(\theta)}=\frac{D_{y}(\theta)}{D_{z}(\theta)}=\frac{\partial \sigma_{y y}^{2}(\theta) / \partial t}{\partial \sigma_{z z}^{2}(\theta) / \partial t}
$$

where the scalar, $C(\theta)$, is the moisture capacity function and the subscript $p$ denotes the $x, y$, or $z$ direction. According to equation (6), the ratio of spatial variances in different directions yields

$$
K_{x}(\theta)=\frac{\partial \sigma_{x x}^{2}(\theta) / \partial t}{\partial \sigma_{z z}^{2}(\theta) / \partial t} K_{z}(\theta) \quad \text { and } \quad K_{y}(\theta)=\frac{\partial \sigma_{y y}^{2}(\theta) / \partial t}{\partial \sigma_{z z}^{2}(\theta) / \partial t} K_{z}(\theta)
$$

10 Note that equations (7) and (8) are independent of the knowledge of the moisture capacity

11 function as long as the function is a scalar as in the Richards' equation. If the moisture capacity

12 function is to be determined, equation (6) then can be used in conjunction with the calculated

13 rate of change of the second spatial moment in the $\mathrm{z}$ direction and $K_{z}(\theta)$ :

$$
\frac{d h}{d \theta}=C(\theta)^{-1}=\frac{1}{2} \frac{\partial \sigma_{z z}^{2}(\theta)}{\partial t} \frac{1}{K_{z}(\theta)}
$$

15 Integration of equation (9) with respect to $\theta$ yields the function describing the relation between $h$ and $\theta$. Equations (5) through (9) are the basic equations for the new method of determining unsaturated hydraulic properties on the basis of observed $\theta$ plumes in a field. 
Our new approach is applied to the field injection experiment (Gee and Ward, 2001).

2 The test facility (Figure 1) was originally designed and developed in 1980s (Sisson and Lu, 3 1984), and will be referred as the S\&L site hereafter. The site mainly consists of sandy deposits

4 with the presence of two layers of distinctly larger fraction of fine sediments (Gee and Ward, 5 2001). Laboratory measurements of moisture retention, saturated and unsaturated conductivity 6 are available for 15 repacked samples from the site (Khaleel and Freeman, 1995; Khaleel et al., 7 1995). The moisture retention data for the laboratory samples for the drainage cycle of up to $8-1000 \mathrm{~cm}$ of pressure head were measured using "Tempe" pressure cells; the rest of the drainage 9 curve up to $-15000 \mathrm{~cm}$ was measured using the pressure plate extraction method (Klute, 1986). 10 A variation of the unit gradient method was used to obtain unsaturated conductivity measurements (Klute and Dirksen, 1986; Khaleel et al., 1995). The best fit curves based on the van Genuchten-Mualem model (Mualem, 1976; van Genuchten, 1980) are shown in Figure 2; they illustrate the general characteristics of hydraulic properties for sediments at the S\&L site. Saturated hydraulic conductivity estimates for small core samples at the S\&L site are also reported in Schaap et al. (2003).

The injection experiments were conducted in June 2000 (Gee and Ward, 2001). Prior to radially arranged cased boreholes (Figure 1). Injections began on June 1st and $4000 \mathrm{~L}$ of water were injected into an injection point (Figure 1) 5-m below the land surface over a 6-hour period. Similarly, $4000 \mathrm{~L}$ of water were injected in each subsequent injection on June 8, June 15, June 22, and June 28. During the experiment, neutron logging in 32 wells took place within a day following each of the first four injections. A wildfire burned close to the test site and prevented immediate logging of the moisture content distribution for the fifth injection. Three additional 
1 readings of the 32 wells were subsequently completed on July 7, 17, and 31. During each

2

3 neutron logging, water contents were monitored at $0.305 \mathrm{~m}$ depth intervals starting from a depth of $3.9 \mathrm{~m}$ and continuing to a depth of $16.8 \mathrm{~m}$. Figures $3 \mathrm{a}, \mathrm{b}, \mathrm{c}, \mathrm{d}, \mathrm{e}$, and $\mathrm{f}$ show cross-sectional views of the 3-D moisture content distribution observed on June 2, June 9, June 16, June 23, July 7, and July 17 (the dotted and solid curves in the figures are discussed later). A geostatistical analysis of the observed moisture plume is reported in Ye et al. (2004; this issue).

Matric potential measurements following the June 15, June 22, and June 28 injections were measured using drive cone tensiometers (Hubbel and Sisson, 1998) at well locations A-3 at a depth of $5.34 \mathrm{~m}$ and H-6 at a depth of $5.28 \mathrm{~m}$ (Figure 1). Although measurements were limited, they do provide some indication about the tension regime (generally between $0.5 \mathrm{~m}$ and $1.5 \mathrm{~m}$ ) in the field during injection and redistribution periods.

\subsection{Estimation of Spatial Moments}

Several investigators analyzed the spatial moment of the observed moisture plume at the field site (e.g., Gee and Ward, 2001; Ye et al., 2004; this issue). A detailed description of the spatial moment analysis and an in-depth discussion of the results used in this paper are given elsewhere (Ye et al., 2004). To limit the number of pages and to maintain the focus of this paper, we present only the Ye et al. analysis results.

\subsection{Estimation of Effective Unsaturated Hydraulic Properties.}

To apply equation (5) to the 2000 injection experiments at the S\&L site, the relation between the calculated first moment $\mathrm{z}_{c}[\mathrm{~m}]$ and time $t$ [d] (Figure 4a) is fitted by a continuous function. For mathematical convenience, we choose a power function

$$
z_{c}=a t^{-b}
$$


1 where $a$ and $b$ are coefficients of the power function and are equal to 8.7723 and 0.0667 ,

2 respectively. The fitted function is shown in Figure $4 \mathrm{a}$ as a solid line. Since the function $V_{z}$ is

3 defined by the rate of change of the first moment in the $z$-direction at measurement time $t_{\mathrm{i}}$,

4

$$
\left.V_{z}\right|_{t_{i}}=\left.\frac{\partial z_{c}}{\partial t}\right|_{t_{i}}
$$

$5 \quad V_{z}$ can be related to the $\theta_{\text {ave }}$ (the average $\theta_{\text {diff }}$ over the volume of the moisture plume that is 6 determined by the dotted ellipsoids shown in Figure 3, see also Ye et al., 2004; this issue) at 7 various measurement times. Assuming that an exponential function describes the relation 8 between $V_{z}$ and $\theta_{\text {ave }}$,

$$
V_{z}=c\left[\exp \left(d \theta_{\text {ave }}\right)\right]
$$

10 we fit (12) to $V_{z}$ and $\theta_{\text {ave }}$ data to obtain coefficients, $c$ and $d$, which are $0.002[\mathrm{~m} / \mathrm{d}]$ and 42.454 ,

11 respectively. The best fit line is shown in Figure $4 \mathrm{~b}$. $\operatorname{Since} \theta=\theta_{\text {ave }}+\theta_{\text {initial }}$, the following 12 equality for $V_{z}$ holds

$$
V_{z}=\frac{d K_{z}}{d \theta}=\frac{d K_{z}}{d \theta_{\text {ave }}}
$$

Integration of equation (13) then yields

$$
K_{z}(\theta)=f\left[\exp \left(g \theta_{\text {ave }}\right)\right]
$$

16 where $f$ and $g$ are coefficients and are equal to $4.72 \times 10^{-5}[\mathrm{~m} / \mathrm{d}]$ and 42.454 , respectively (the

17 average $\theta_{\text {initial }}$ was estimated to be 0.0846 for the entire field site). 
The spatial variances as a function of time for the observed moisture plume are shown in

2 Figures $5 \mathrm{a}, \mathrm{b}$ and $\mathrm{c}$. A second-order polynomial function in $t$ is fitted (solid lines in Figures $5 \mathrm{a}$,

$3 \mathrm{~b}$, and c) to the spatial variances of the observed data for the entire injection experiment period.

4 Differentiation of the fitted function and evaluation for the day measurements were taken then

5 yields the rate of change in the spatial variance. These rates of change in $\sigma_{x x}^{2}$ and $\sigma_{y y}^{2}$ are then

6 related to the average $\theta_{\text {ave }}$ corresponding to each day, again assuming that a second order

7 polynomial is adequate. Once the ratios of $K_{x}(\theta)$ and $K_{y}(\theta)$ to $K_{z}(\theta)$ are known, $K_{x}(\theta)$ and $K_{y}(\theta)$

8 are evaluated using $K_{z}(\theta)$ from equation (14) (units are $\mathrm{m} / \mathrm{d}$ ). These are:

$$
\begin{aligned}
& K_{z}(\theta)=f \exp \left[g\left(\theta-\theta_{\text {initial }}\right)\right] ; \quad f=4.72 \times 10^{-5} \mathrm{~m} / d ; g=42.45 ; \quad \theta_{\text {initial }}=0.0846 \\
& K_{x}(\theta)=\left(a_{1} \theta^{2}-a_{2} \theta+a_{3}\right) K_{z}(\theta) ; \quad a_{1}=988.48 ; \quad a_{2}=338.78 ; \quad a_{3}=30.60 \\
& K_{y}(\theta)=\left(b_{1} \theta^{2}-b_{2} \theta+b_{3}\right) K_{z}(\theta) ; \quad b_{1}=693.11 ; \quad b_{2}=237.55 ; \quad b_{3}=22.14 .
\end{aligned}
$$

10 These unsaturated hydraulic conductivity functions are plotted in Figure 6 as a function of $\theta$.

11 Notice that during the analysis, the $x, y$, and $z$ coordinates were assumed to be the principal

12 directions of the effective hydraulic conductivity tensor. That is, the off-diagonal terms in the

13 hydraulic conductivity tensor are assumed to be zero.

14 For the same equivalent homogeneous medium, we can also obtain the moisture retention

15 curve ( $\theta-h$ relation) as follows. We first substitute the fitted second-order polynomial relation

16 between $\sigma_{z z}^{2}$ and time $t$, and the estimate of $K_{z}(\theta)$ from equation (15) into equation (9) to yield

$$
\begin{aligned}
\frac{\partial h}{\partial \theta} & =\frac{1}{2 K_{z}(\theta)} \frac{\partial \sigma_{z z}^{2}}{\partial t}=c_{1} t \exp (-g \theta)+c_{2} \exp (-g \theta) \\
c_{1} & =-18.9 ; c_{2}=951.3 ; g=42.45 .
\end{aligned}
$$

18 Since $\theta=\theta_{\text {diff }}+\theta_{\text {initial, }}$, a regression analysis of $\theta_{\text {diff }}$ and $t$ data yields (see Figure 7)

$$
\begin{aligned}
& t=d_{1} \exp \left\{-d_{2}\left(\theta-\theta_{\text {initial }}\right)\right\} \\
& d_{1}=202.45 ; \quad d_{2}=39.803 ; \quad \theta_{\text {initial }}=0.0846
\end{aligned}
$$


1 Substituting equation (17) into equation (16), and integrating equation (16) with respect to $2 \quad \theta$ yields

3

4

5 the condition $|h|=1.2 \mathrm{~m}$ at $\theta=0.0846$ in equation (18), $\mathrm{C}=0.406$. This estimated relation between

$6 h$ and $\theta$ is shown in Figure 8, which shows a generally decreasing $\theta$ as $h$ becomes more negative.

7 We can also estimate the moisture retention function for the equivalent homogeneous medium

where $\mathrm{h}$ is negative, || represents the absolute value, and $\mathrm{C}$ is the constant of integration. Using based on measurements for the 15 core samples (Figure 2a). First, we assume that the effective moisture retention function is described by van Genuchten's (1980) model:

$$
\theta(h)=\left(\theta_{s}-\theta_{r}\right)\left(1+\gamma|h|^{\eta}\right)^{-m}+\theta_{r}
$$

where $\|$ is the absolute value, $\theta_{s}$ is the saturated moisture content, $\theta_{r}$ is the moisture content at residual saturation and $\gamma, \eta$, and $m$ are curve-fitting parameters with $m=1-1 / \eta$. Fitting equation (19) to obtain an average of the measured retention data for 15 samples (Figure 2a) yields 0.304 , $0.00726,25.054 \mathrm{~m}^{-1}$, and 1.3702 for $\theta_{s}, \theta_{r}, \gamma$, and $\eta$, respectively. A plot of the average $\theta(h)$ function is also shown in Figure 8. A comparison of the estimated $\theta(h)$ using our new approach and that based on the core samples indicates that the estimated function generally agrees well with the average $\theta(h)$ function based on the 15 core samples. Nonetheless, we decided to use the average $\theta(h)$ based on the core samples as the $\theta(h)$ function for the equivalent homogeneous medium.

Using the value of $\theta_{s}$ from the average $\theta(h)$ function and equation (15), the saturated hydraulic conductivities $\left(K s_{x}, K s_{y}\right.$, and $\left.K s_{z}\right)$ of the equivalent homogeneous medium for the $\mathrm{S} \& \mathrm{~L}$ site are estimated to be $9.9066 \mathrm{~m} / \mathrm{d}, 7.302 \mathrm{~m} / \mathrm{d}$, and $0.5224 \mathrm{~m} / \mathrm{d}$, respectively.

$$
\begin{aligned}
& |h|=e_{1} \exp \left(-e_{2} \theta\right)-e_{3} \exp (-g \theta)+C \\
& e_{1}=1141.22 ; e_{2}=82.253 ; e_{3}=22.41 ; g=42.45
\end{aligned}
$$




\subsection{Assessment of the Estimated Effective Hydraulic Properties.}

The moisture movement during the 2000 injection experiment at the S\&L site was simulated numerically using the effective unsaturated hydraulic conductivities and moisture retention curve derived from the preceding analysis. Since the medium is assumed to be homogeneous and only a point injection is involved, a two-dimensional (2-D) finite element variably saturated flow and transport model (VSAFT2, Yeh et al., 1993; available at www.hwr.arizona.edu/yeh) was used to simulate the 3-D axisymmetric infiltration problem. The model solves a mixed form of the Richards' equation in radial coordinates:

$$
\omega S_{s} \frac{\partial h}{\partial t}+\frac{\partial \theta}{\partial t}=\frac{1}{r} \frac{\partial}{\partial r}\left[\bar{K}_{r}(h) r \frac{\partial h}{\partial r}\right]+\frac{\partial}{\partial z}\left[\bar{K}_{z}(h) \frac{\partial(h+z)}{\partial z}\right],
$$

where $t$ is time, $r$ is the radial distance from the center of the injection point, and $z$ is the positive upward vertical coordinate. The pressure head, $h$, is positive when the medium is fully saturated and negative when the medium is partially saturated. The term $S_{s}$ represents specific storage (assumed to be $0.00001 \mathrm{~m}^{-1}$ for the $\mathrm{S} \& \mathrm{~L}$ site), which is used to account for possible saturation near the injection well. The transitioning parameter, $\omega$, is equal to one when $h$ is greater than or equal to zero, and zero when $h$ is negative. The unsaturated hydraulic conductivity functions, $\bar{K}_{r}(h)$ and $\bar{K}_{z}(h)$, are based on the calculated $K_{x}(\theta)$ and $K_{z}(\theta)$ (equation 15), assuming $K_{x}(\theta)=K_{y}(\theta)$; the moisture retention curve is described by equation (19). VSAFT2 solves equation (20) using a Newton-Raphson (N-R) iteration scheme to avoid mass balance issues.

The numerical simulation considers a 2-D domain of $15 \mathrm{~m}$ (horizontal) $\times 15 \mathrm{~m}$ (vertical). The left boundary of the domain was aligned with the center of the injection well. The domain 
1 was discretized into 900 uniform elements of size $0.5 \mathrm{~m} \times 0.5 \mathrm{~m}$ in $r$ and $z$. The other three sides

2 of the domain were prescribed as constant head boundaries of a pressure head equal to $-1.2 \mathrm{~m}$,

3 corresponding to the initial pressure head. Three nodes along the left boundary at an elevation of

$4 \quad 9.5 \mathrm{~m}$ to $10.5 \mathrm{~m}$ were assigned as flux boundary nodes, where the injection rate and its schedule

5 were specified according to measurements (Gee and Ward, 2001).

7 4. RESULTS AND DISCUSSION

Figure 6 presents a comparison of the estimated $K(\theta)$, using the newly-developed spatial moment-based technique, with measured unsaturated hydraulic conductivities (Khaleel and Freeman, 1995; Khaleel et al., 1995) and saturated hydraulic conductivities (Schaap et al., 2003) for small-scale core samples. All our effective $K(\theta)$ estimates fall within the cluster of the measured conductivity values, suggesting that the estimates are reasonable - especially since they are derived independent of the conductivity measurements. The $K_{x}(\theta)$ and $K_{y}(\theta)$ estimates are shown to be greater than the $K_{z}(\theta)$ estimates, indicating greater anisotropy in the $x-z$ and $y-z$ planes. Only small anisotropy exists in the $x-y$ plane as reflected by the small differences between the effective $K_{x}(\theta)$ and $K_{y}(\theta)$ estimates. To our knowledge, this is the first quantitative estimate of 3-D moisture-dependent anisotropy in unsaturated hydraulic conductivity under field conditions.

As shown in Figure 6, the rate of reduction in $\ln K_{z}(\theta)$ is constant when $\theta$ decreases. The rates of reduction in $\ln K_{x}(\theta)$ and $\ln K_{y}(\theta)$ are greater than for $\ln K_{z}(\theta)$ for $\theta$ values larger than 0.2 ; they become similar to that of $\ln K_{z}(\theta)$ (i.e., the approximate parallel lines in Figure 6 ) in a narrow range of $\theta$ of about 0.2 . The rate of reduction in $\ln K_{x}(\theta)$ and $\ln K_{y}(\theta)$ becomes smaller than that in $\ln K_{z}(\theta)$ when the $\theta$ values are less than 0.2 . Such $K_{x}(\theta), K_{y}(\theta)$, and $K_{z}(\theta)$ functions 
1 imply a moisture-dependent anisotropy ratio function (i.e., $K_{x}(\theta) / K_{z}(\theta)$, or $K_{y}(\theta) / K_{z}(\theta)$ ) of a

2 concave shape. That is, the anisotropy decreases first and then increases as soil becomes less

3 saturated. A concave-shaped moisture-dependent anisotropy, as explained by the stochastic

4 analysis (Yeh et al., 1985b and c), is the result of a stratified geological formation consisting of

5 layers whose saturated hydraulic conductivity is correlated with a pore-size distribution

6 parameter (i.e., the exponent in the Gardner exponential unsaturated hydraulic conductivity

7 model; Gardner, 1958). Therefore, the estimated concave moisture-dependent anisotropy is

8 consistent with findings about the geology in Gee and Ward (2001) and (Ye et al., 2004; this

9 issue); the S\&L site is composed of alternating layers of coarse- and fine-textured sands (see also 10 the hydraulic properties in Figure 2).

11 Cross-sectional views of the simulated moisture plume are shown in Figure 9 for six observation days. The mass centers and spatial variances of the simulated plume are compared with those of the observed plume in Figure 10. In general, the simulated moisture plumes mimic the observed plumes but differ in detail. Likewise, in general, the spatial moments of the simulated plume are similar to those of the observed plume but different in detail.

The discrepancy in details between the observed and simulated plumes can be explained by the moisture diffusivity length [L] concept. It is defined as

$$
\lambda(\theta)=\frac{D(\theta)}{d K(\theta) / d \theta}
$$

which is analogous to the dispersivity [L] in solute transport analysis for porous media. While the dispersivity represents the distance over which mixing in porous media is effective, the moisture diffusivity length denotes the distance over which capillary forces are active. The calculated moisture diffusivity lengths are shown in Figure 11 as a function of $\theta$ for the 15 core samples from the S\&L site. Note that the moisture diffusivity concept is not valid for pressure 
1 heads greater than the air entry pressure head and for $\theta$ lower than the residual $\theta$. Figure 11

2 shows that near saturation, the moisture diffusivity length is large; it decreases as the medium

3 becomes less saturated; and it then increases near the residual water content. For the range of

$4 \theta$ values ( 0.10 to 0.25$)$ observed during the injection experiment, the moisture diffusivity lengths

5 are less than the vertical correlation length scale of heterogeneity (about $3.5 \mathrm{~m}$; Ye et al., 2004;

6 this issue) at the field site. The capillary forces thus cannot overcome the dominant effect of

7 heterogeneity (layers) and REVs for the moisture content during the experiment do not exist.

8 The simulated moisture plume using the equivalent homogeneous medium approach

9 consequently only represents the ensemble average distribution - not single realizations (i.e., the

10 observed moisture plume) - a finding similar to that of Thierrin and Kitanidis (1994) for solute

11 plumes in saturated media. In spite of discrepancies in the details of moisture content

12 distribution, the spatial moments of the simulated plume, based on the newly-developed effective

13 parameter approach, apparently reproduce the spatial moments of the observed plume reasonably

14 well.

Ellipsoids (solid lines in Figure 3) showing the average location and dimension of the

16 plume are determined using an approach similar to that of Ye et al. (2004) on the basis of spatial

17 moments of the simulated plume at different times. The mass center of these ellipsoids are at the

18 same coordinates as that of the injection well. Compared with the actual plumes and the dotted

19 ellipses based on moment calculations from the observed data, the new ellipses define

20 remarkably well the spatial variation of the moisture plumes. However, they overestimate the

21 spatial variation in the $z$ direction during the redistribution period and cannot reproduce the

22 dipping of the ellipses calculated on the basis of moments of the observed moisture plume.

23 Apparently, had we not assumed that the $x$ and $z$ directions are the principal directions of the 
1 effective hydraulic conductivity tensor (i.e., the mixed terms in the tensor were considered), the

2 discrepancies would have been minimized. An omission of variation of the principal directions

3 (see Ye et al., 2004) in our analysis may also have exacerbated the discrepancies.

The simulation also replicates the observed split of peaks of the moisture plume (compare Figures $3 a$ and 9a) during the first single injection experiment. Physically, the observed split of the moisture plume is attributed to the fine- and coarse-textured layered structures at the field site (Ye et al., 2004; this issue). In this equivalent homogeneous medium, the concave shape of anisotropy in the effective unsaturated hydraulic conductivity is responsible for the split of the simulated plume. Specifically, during the injection, the region adjacent to the injection point is near saturation; its horizontal hydraulic conductivity is larger than the vertical; and the injected water spreads out laterally. As water spreads out laterally, the medium near the injection point becomes less saturated and its horizontal hydraulic conductivity approaches the concave portion of the effective conductivity, where the medium becomes less anisotropic. Water thus moves more vertically than horizontally due to a greater hydraulic gradient in the vertical direction. As the water migrates downward, it encounters dry regions of the medium, whose horizontal conductivity is greater than the vertical and as such spreads out laterally again. This concave anisotropy in an equivalent homogeneous medium certainly portrays the bulk geologic setting at the field site. Nonetheless, the ensemble concept embedded in the effective conductivity approach does not allow a correct prediction of the location (layer interface) where the split actually occurred in the field (i.e., again because of a single realization).

Several assumptions and approximations behind our new approach may have led to discrepancies in the spatial moments of the simulated and observed plumes as well as in the detailed shape of the plumes. First, the moment analysis of the data implicitly assumes a 
1 unimodal distribution of the $\theta_{\text {diff }}$ even though the moisture plume as shown in Figure 3 exhibits a

2 bimodal distribution. The moments calculated on the basis of unimodal distribution simply

3 represent the averaged moments. This approximation is identical to the one used in spatial

4 moment analysis and in macrodispersion concept (Sudicky, 1986; Barry and Sposito. 1990;

5 Garabedian et al., 1991; Adams and Gelhar, 1992) for tracer plumes at the Borden, Cape Cod,

6 and Mississippi tracer experiments. It is also identical to the normality assumption used in many

7 statistical analyses. Our approach predicts the ensemble or bulk moments of the moisture

8 plume, and therefore the ensemble-averaged behavior of the plume.

9 Our approach implicitly assumes a single impulse injection of water while multiple 10 injections were conducted during the experiment. Nonetheless, the fine-textured layer right 11 below the injection point may have acted as a buffer that slowly released the injected water.

12 Thus, for the overall moisture plume, multiple injections may be approximated as a single 13 source.

14 We approximate the $\theta_{\text {ave }}$ in equation (11) as the difference between the spatially averaged $15 \theta$ at a given time and that of the initial moisture content. In theory, this value should have been 16 evaluated at the center of mass of the moisture plume. Moisture diffusivity in equations (6) and 17 (9) similarly should have been evaluated at the $\theta$ value that represents the average of all $\theta$ values located at the square-root of the spatial variance. The principal directions of the effective hydraulic conductivity are further assumed to be aligned with the $x, y$, and $z$ coordinates and remain fixed. In order to better reproduce the dynamically varying principal directions of the

21 moisture plume (Ye et al., 2004), variations in the principal directions of the unsaturated 22 hydraulic conductivity tensor should be considered as the moisture plume migrates. Lastly, as 
1 suggested by the work of McCord et al. (1991) and Mantoglou and Gelhar (1987), we also

2 neglect large-scale hysteresis in the effective unsaturated hydraulic conductivities.

3 In spite of these assumptions and approximations, we find that the effective $K$ estimates

4 agree mostly within an order of magnitude of measurements based on the core samples (Figure

5 6). Furthermore, the estimated anisotropy in $K$ appears to reproduce the observed $\theta$ field

6 reasonably well. This is an important result, considering the fact that very few field studies and

7 techniques are available for evaluating moisture-dependent anisotropy. Our new approach

8 estimates the effective $K$ solely based on the temporal evolution (snapshots) of spatial moments

9 of moisture plumes. Neither our prior knowledge on the hydraulic conductivity of the core

10 samples nor any geological structure information is used during the estimation.

11 Lastly, in the above analysis we chose to use power, exponential, and polynomial

12 functions for fitting the moments of the observed plume. While this approach served our

13 purpose, alternate innovative approaches are possible. For example, one can follow the work of

14 Yeh et al. (1985a, b, and c), Mantoglou and Gelhar (1987) and Polmann et al. (1991) on the use

15 the pressure-based Richards' equation at the local scale to derive a theoretical relation between

16 the spatial moments of a moisture plume and spatial statistics of local-scale unsaturated

17 hydraulic properties (similar to the stochastic macrodispersion analysis of Dagan, 1987) under

18 radial flow conditions. Instead of the power or polynomial models, these relations can be fitted

19 to the observed moments. The effective $K(\theta)$ that fits the spatial moment data can then be

20 derived, and the spatial statistics of small-scale hydraulic properties also can be inferred. In turn,

21 the theoretical effective $\theta(h)$ can be determined. This suggested approach is of great practical

22 significance. For example, if the spatio-temporal evolution of a moisture plume in the vadose

23 zone can be monitored by remote sensing or geophysical surveys such as electrical resistivity 
1 tomography (ERT) (Yeh et al., 2002), integrative ERT (Liu and Yeh, 2004) or cross bore hole

2 ground-penetrating radar (GPR) (Binley et al., 2002; Grote et al., 2003; Alumbaugh et al., 2002),

3 then the effective unsaturated hydraulic properties and spatial variability of the vadose zone can

4 be estimated with relative ease. The estimated properties based on moisture plumes then can be 5 used in a predictive model for water resources management or for planning purposes for a large6 scale vadose zone.

\section{SUMMARY AND CONCLUDING REMARKS}

A new and practical technique, based on the $\theta$-based Richards' equation and evolution of 10 spatial moments of a moisture plume, was developed to estimate the effective unsaturated 11 hydraulic conductivity tensor of an equivalent homogeneous medium. The effective hydraulic conductivities, based on the new approach and analysis of the injection experiment data at the

13 S\&L site, compare well with the laboratory-measured conductivities for core samples. 14 Furthermore, spatial moments of the simulated plume based on the effective hydraulic 15 conductivities are in good agreement with those for the observed plume. The new technique not 16 only provides a new way to estimate effective $K$ but also allows the previously developed 17 moisture-dependent anisotropy concept to be quantitatively evaluated. The overall good agreement of conductivities derived from laboratory measurements and the good comparison between the numerically simulated plume and the observed plume demonstrate that moisturedependent anisotropy is a valid, reproducible phenomenon in the field. The macroscopic anisotropy does indeed vary with decreasing moisture content of the unsaturated medium. We

22 illustrate the fact that, at this particular field site, the effective hydraulic properties of an equivalent homogeneous medium can yield a similar temporal evolution of spatial moment of the 
1 observed moisture plume. The simulated moisture plume, based on the effective hydraulic 2 conductivities of an equivalent homogeneous medium, however, does not necessarily resemble 3 the observed distribution. We further show that the relation between the moisture diffusivity 4 length and the vertical correlation scale of the dominant heterogeneity may ultimately determine 5 the correspondence between the observed and simulated plumes using the effective property 6 approach. Nonetheless, in spite of the inherent limitation of the effective property approach, we 7 feel that the new technique is a useful practical tool for estimating effective unsaturated 8 hydraulic conductivities based on snapshots of moisture movement in a large-scale vadose zone. 9 It is relatively simple and applicable to column-, sandbox-, or field-scale problems. 
1

2

3

4

5

\section{ACKNOWLEDGMENTS}

The injection site experiments in the year 2000 were conducted as part of the Vadose Zone Transport Field Study at the Hanford Site; Glendon Gee of Pacific Northwest National Laboratory (PNNL) was the project manager. Anderson Ward of PNNL provided the moisture content data. J. Buck Sisson (formerly of Rockwell Hanford Operations, Richland, WA) originally designed the injection test site layout and conducted the first experiments at the site in 1980. The work reported was performed for the CH2M Hill Hanford Group Inc. and the U.S. Department of Energy under Contract DE-AC06-99RL14047. The first author was funded in part by NSF/SERDP grant \#EAR-0229717. We express our gratitude to Alexandre Desbarats who suggested evaluating the first moment of the moisture plume and Art Warrick with whom the first author frequently explores new ideas with during coffee and golf breaks. Many thanks are also extended to the anonymous $\mathrm{AE}$ and reviewers who provided constructive suggestions. In particular, the $\mathrm{AE}$ provided very insightful suggestions. Reference herein to any specific commercial product, process, or service by trade name, trademark, manufacturer, or otherwise, does not necessarily constitute or imply its endorsement, recommendation, or favoring by the United States Government or any agency thereof or its contractors or subcontractors. The views and opinions of the authors do not necessarily state or reflect those of the United States Government or any agency thereof. 


\section{REFERENCES}

Ababou, R., Three-dimensional flow in random porous media, Ph.D. thesis, Mass. Inst. of Technol., Cambridge, Mass., 1988.

Adams, E. E. and L. W. Gelhar, Field study of dispersion in a heterogeneous aquifer, 2, spatial moments analysis, Water Resour. Res., 28(12), 1992.

Alumbaugh, D., P. Y. Chang, L. Paprocki, J. R. Brainard, R. J. Glass, and C. A. Rautman, Estimating moisture contents in the vadose zone using cross-borehole ground penetrating radar: A study of accuracy and repeatability, Water Resour. Res., 38(12), 1309, doi:10.1029/2001WR000754, 2002.

Aris, R., On the dispersion of a solute in a fluid flowing through a tube, Proc. R. Soc. London, Ser. A, 235, 67-78, 1956.

Barry, D. A., and G. Sposito, Three-dimensional statistical moment analysis of the Stanford/Waterloo Borden tracer data, Water Resour. Res. 26(8): 1735-1747, 1990.

Binley, A., P. Winsip and R. Middleton, High-resolution characterization of vadose zone dynamics using cross-borehole radar, Water Resour. Res. 37(11): 2639-2652, 2001.

Csanady, G. T., Turbulent diffusion in the environment, D. Reidel Publish Company, 248 pp, 1973.

Dagan, G., Theory of solute transport by groundwater, Ann. Rev. Fluid Mech., 19, 183-215, 1987.

de Marsily, G., “Quantitative Hydrogeology, Groundwater Hydrology for Engineers”, Academic Press, Inc., pp. 440, 1986.

Desbarats, A. J., Scaling of constitutive relationships in unsaturated heterogeneous media, Water Resour. Res., 34 (6), 1427-1436, 1998. 
1 Fischer H. B., E. J. List, R. C.Y. Koh, J. Imberger, and N. H. Brooks, Mixing in Inland and

$2 \quad$ Costal Waters, pp. 483, Academic Press, 1979.

3 Freyberg, D. L., A natural gradient experiment on solute transport in a sandy aquifer, 2. Spatial

4 moments and the advection and dispersion of noncreative tracers, Water Resour. Res. 22(13):

5 2031-2046, 1986.

6 Garabedian, S.P. D.R. Leblanc, L.W. Gelhar and M. A. Celia., Large-scale natural gradient

7 tracer test in sand and gravel, Cape Cod, Massachusetts, 2, Analysis of spatial moments for a

$8 \quad$ nonreactive tracer, Water Resour. Res. 27 (5), 911-924, 1991.

9 Gardner, W. R., Some steady state solutions of unsaturated moisture flow equations with 10 application to evaporation from a water table, Soil Sci., 85, 228-232, 1958.

11 Gee, G. W., and A. L. Ward, Vadose Zone Transport Field Study: Status Report, PNNL-13679, 12 Pacific Northwest National Laboratory, Richland, WA, 2001.

13 Gelhar, L. W., Stochastic Subsurface Hydrology, 390p, Prentice Hall, Englewood Cliffs, N. J., $14 \quad 1993$.

15 Green, T. R., and D. L. Freyberg, State-dependent anisotropy: Comparisons of quasi-analytical 16 solutions with stochastic results for steady gravity drainage, Water Resour. Res., 31(9), 2201$17 \quad 2212,1995$.

Greenholtz, D. E., T.-C. J. Yeh, M. S. B. Nash, and P. J. Wierenga, Spatial variability of soil water tension, soil water content and unsaturated hydraulic conductivity, Journal of Contaminant Hydrology, 3, 227-250, 1988.

21 Grote, K., S. Hubbard, and Y. Rubin, Field-scale estimation of volumetric water content using 22 ground-penetrating radar ground wave techniques, Water Resour. Res., 39(11), 1321, 23 doi:10.1029/2003wr002045, 2003 
1 Holt, R. M., J. L. Wilson, and R. J. Glass, Spatial bias in field-estimated unsaturated hydraulic

2 properties, Water Resour. Res., 38(12), 1311, doi:10.1029/2002WR001336, 2002.

3 Hubbell, J.M. and J.B. Sisson, Advanced tensiometer for shallow or deep soil water potential

$4 \quad$ measurements. Soil Sci. 163(4): 271-276, 1998.

5 Khaleel, R. and E. J. Freeman, Variability and Scaling of Hydraulic Properties for 200 Areas

6 Soils, Hanford Site, Westinghouse Hanford Company, Richland, WA, 1995.

7 Khaleel, R., J. F. Relyea, and J. L. Conca, Evaluation of van Genuchten-Mualem relationships to

8 estimate unsaturated conductivity at low water contents, Water Resour. Res., 31 (11), 2659-

$9 \quad 2668,1995$.

10 Khaleel, R., T.-C. J. Yeh, and Z. Lu, Upscaled flow and transport properties for heterogeneous

11 unsaturated media, Water Resour. Res., 38 (5), 1053, doi:10.1029/2000WR000072, 2002.

12 Klute, A. 1986. Water Retention: Laboratory Methods, in Methods of Soils Analysis, Part I, 13 edited by A. Klute, pp. 635-660. Amer. Soc. of Agron. Madison, Wisc.

14 Klute, A., and C. Dirksen, Hydraulic Conductivity and Diffusivity: Laboratory Methods, in 15 Methods of Soil Analysis, Part I, edited by A. Klute, pp. 687-734, Am. Soc. Agron., Madison, 16 Wisc, 1986.

17 Liu, S., and T. -C. J. Yeh, An integrative approach for monitoring water movement in the 18 vadose zone, to appear in Vadose Zone Journal, 2004.

19 Lu, A. and R. Khaleel, Calibration/Validation of VAM3D Model Using Injection Test Data at 20 Hanford, in Vadose Zone Modeling Workshop Proceedings, March 29-30, 1993. R. Khaleel 21 (ed.), WHC-MR-0420, Westinghouse Hanford Company, Richland, WA, 1993.

22 Mantoglou, A., and L. W. Gelhar, Stochastic modeling of large-scale transient unsaturated flow, 23 Water Resour. Res., 23 (1), 37-46,1987. 
1 McCord, J. T., D. B. Stephens, and J. L. Wilson, Hysteresis and state dependent anisotropy in 2 modelling unsaturated hillslope hydrologic processes, Water Resour. Res., 27(7), 1501- 1518, $3 \quad 1991$.

4 Mualem, Y., A new model for predicting the hydraulic conductivity of unsaturated porous 5 media, Water Resour. Res., 12, 513- 522, 1976.

6 Polmann, D. J., D. Mclaughlin, S. Luis, L. W. Gelhar, R. Ababou, Stochastic modeling of large7 scale flow in heterogeneous unsaturated soils, Water Resour. Res. 27 (7), 1447-1458, 1991.

8 Rudolph, D. L., R. G. Kachanoski, M. A. Celia, D. R. LeBlanc, and J. H. Stevens, Infiltration 9 and solute transport experiments in unsaturated sand and gravel, Cape Cod, Massachusetts: Experimental design and overview of results, Water Resour. Res. 32(3), 519-532, 1996.

Schaap, M.G., P.J. Shouse, and P.D. Meyer, Laboratory measurements of the unsaturated hydraulic properties at the vadose zone transport field site, PNNL-14284, Pacific Northwest National Laboratory, Richland, WA, 2003.

Sisson, J.B., and A.H. Lu., Field Calibration of Computer Models for Application to Buried Liquid Discharges: A Status Report. RHO-ST-46P. Rockwell Hanford Operations, Richland, Washington, 1984.

Stephens, D. B., and S. Heermann, Dependence of anisotropy on saturation in a stratified sand, Water Resour. Res., 24 (5), 770- 778, 1988.

Sudicky, E. A., A natural gradient experiment on solute transport in a sand aquifer: Spatial variability of hydraulic conductivity and its role in the dispersion process, Water Resour. Res. 22 (13), 2069-2082, 1986.

Thierrin, J. and P. K. Kitanidis, Solute dilution at the Borden and Cape Cod groundwater tracer tests, Water Resour. Res. 30(11), 2883-2890, 1994. 
van Genuchten, M. Th., A closed-form solution for predicting the conductivity of unsaturated soils, Soil Sci. Soc. Am. J., 44, 892-898,1980.

Wierenga, P.J., D.B. Hudson, and M.R. Kirkland, The second Las Cruces trench experiment: experimental results and two-dimensional flow predictions, Water Resour. Res. 27 (10), 2707$2718,1991 b$.

Wierenga, P.J., R.G. Hills, and D.B. Hudson, The Las Cruces trench site: characterization, experimental results, and one-dimensional flow predictions, Water Resour. Res. 27(10), 26952705, 1991a.

Wildenschild, D., and K. H. Jensen, Laboratory investigations of effective flow behavior in unsaturated heterogeneous sands, Water Resour. Res., 35 (1), 17-28, 1999a.

Wildenschild, D., and K. H. Jensen, Numerical modeling of observed effective flow behavior in unsaturated heterogeneous sands, Water Resour. Res., 35 (1), 29-42, $1999 \mathrm{~b}$.

Ye, M., R. Khaleel, and T.-C. J. Yeh, Stochastic analysis of moisture plume dynamics of a field injection experiment, Water Resour. Res (this issue), 2004.

Yeh, T.-C. J., and D. J. Harvey, Effective unsaturated hydraulic conductivity of layered sands, Water Resour. Res., 26(6), 1271-1279, 1990.

Yeh, T.-C. J., L. W. Gelhar, and A. L. Gutjahr. 1985a. Stochastic analysis of unsaturated flow in heterogeneous soils: 1, statistically isotropic media, Water Resour. Res. 21(4), 447-456.

Yeh, T.-C. J., L. W. Gelhar, and A. L. Gutjahr. 1985b. Stochastic analysis of unsaturated flow in heterogeneous soils: 2 , statistically anisotropic media, Water Resour. Res. 21(4), 457-464.

Yeh, T.-C. J., L. W. Gelhar, and A. L. Gutjahr. 1985c. Stochastic analysis of unsaturated flow in heterogeneous soils: 3, observations and applications, Water Resour. Res. 21(4), 465-471.

Yeh, T.-C. J., One-dimensional steady-state infiltration in heterogeneous soils, Water Resour. 
$1 \quad$ Res., 25(10), 2149-2158, 1989.

2 Yeh, T.-C. J., R. Srivastava, A. Guzman, and T. Harter, A numerical model for two dimensional 3 flow and chemical transport, Groundwater, 31(4), 634-644, 1993.

4 Yeh, T.-C. J., S. Liu, R. J. Glass, K. Baker, J.R. Brainard, D. Alumbaugh, and D. LaBrecque, A 5 geostatistically based inverse model for electrical resistivity surveys and its applications to 6 vadose zone hydrology, Water Resour. Res., 38(12), 1278, doi: 10.1029/2001WR001204, 72002. 


\section{LIST OF FIGURES}

2 Figure 1. Plan view of the Sisson and Lu injection test site (1984) and well numbering scheme

3 (after Gee and Ward, 2001).

4 Figure 2. Fitted van Genuchten-Mualem moisture retention and unsaturated conductivity curves

5 for 15 samples.

6 Figure 3. Snapshots of the observed moisture plumes and the ellipsoids (dotted, based on the

7 observed plume and solid, the simulated plume).

8 Figure 4. a) Mass centers in the $\mathrm{z}$ direction on different days and the best-fit function; $\mathrm{b}) \mathrm{V}_{\mathrm{z}}$ as a

9 function of $\theta_{\text {ave }}$ and the best-fit function for the estimated unsaturated hydraulic conductivity in

10 the $\mathrm{z}$ direction.

11 Figure 5. The spatial variances of the observed moisture plume on different days and the best-fit

12 functions describing evolution of the variances as a function of time in the $\mathrm{x}, \mathrm{y}$, and $\mathrm{z}$ directions

13 (a, b, and c, respectively).

14 Figure 6. Estimated hydraulic conductivity versus average moisture content using our new

15 approach. Measured hydraulic conductivity values from core samples are presented as circles.

16 Figure 7. Relation between $t$ and $\theta_{\text {diff. }}$.

17 Figure 8. Plot of $h$ versus $\theta$ estimated from the new approach and those from an average of 15

18 samples.

19 Figure 9. Simulated moisture plume using the effective hydraulic properties based on our new

20 method.

21 Figure 10. Comparison of mass centers and spatial variances of the simulated (dashed) and

22 observed (solid) moisture plumes.

23 Figure 11. Water diffusivity length and moisture content relations for the 15 soil samples. 


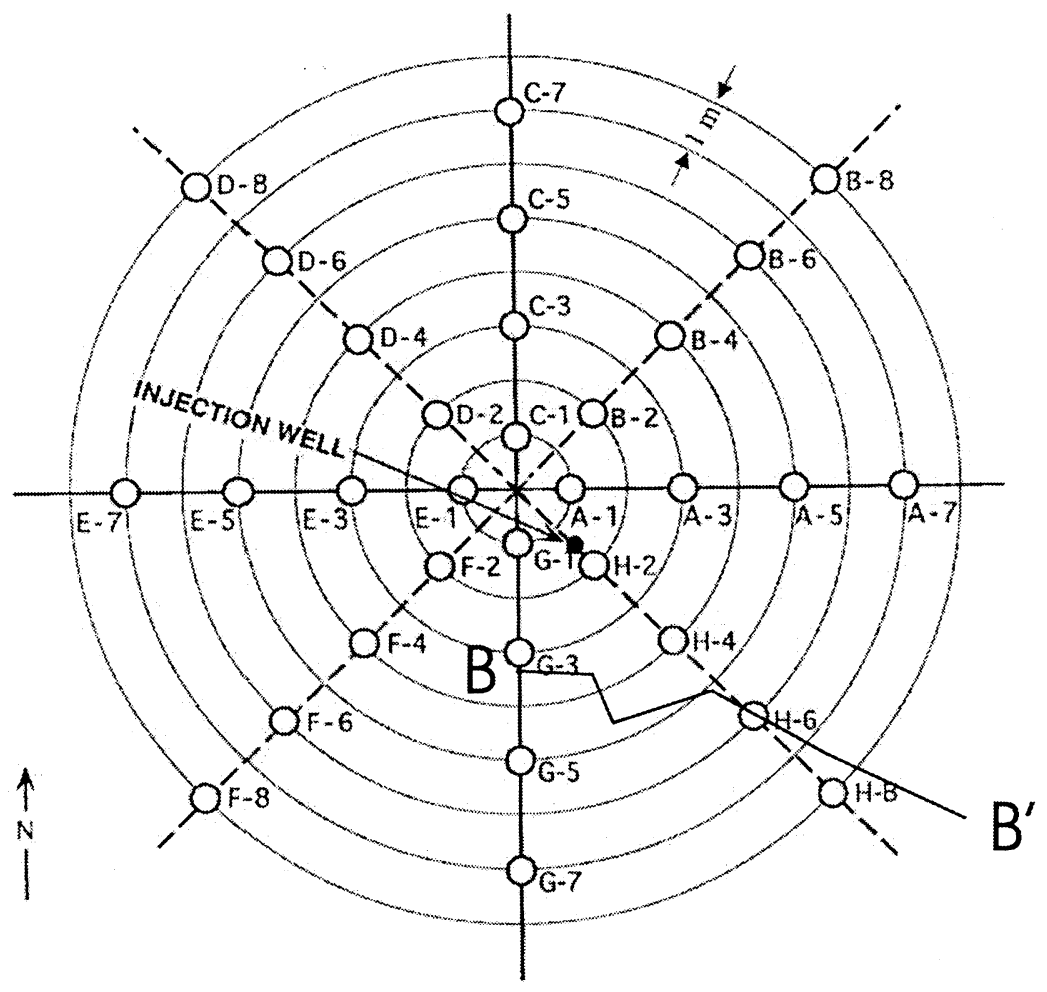

Figure 1. Plan view of the Sisson and Lu injection test site (1984) and well numbering scheme (after Gee and Ward, 2001). 
a)

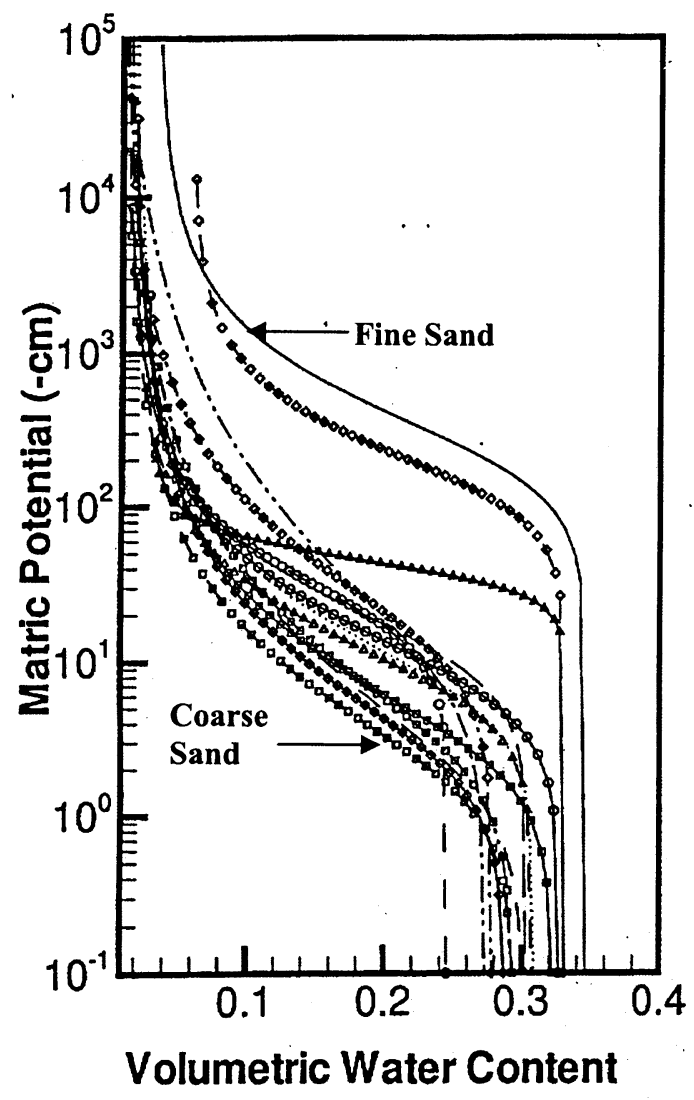

b)

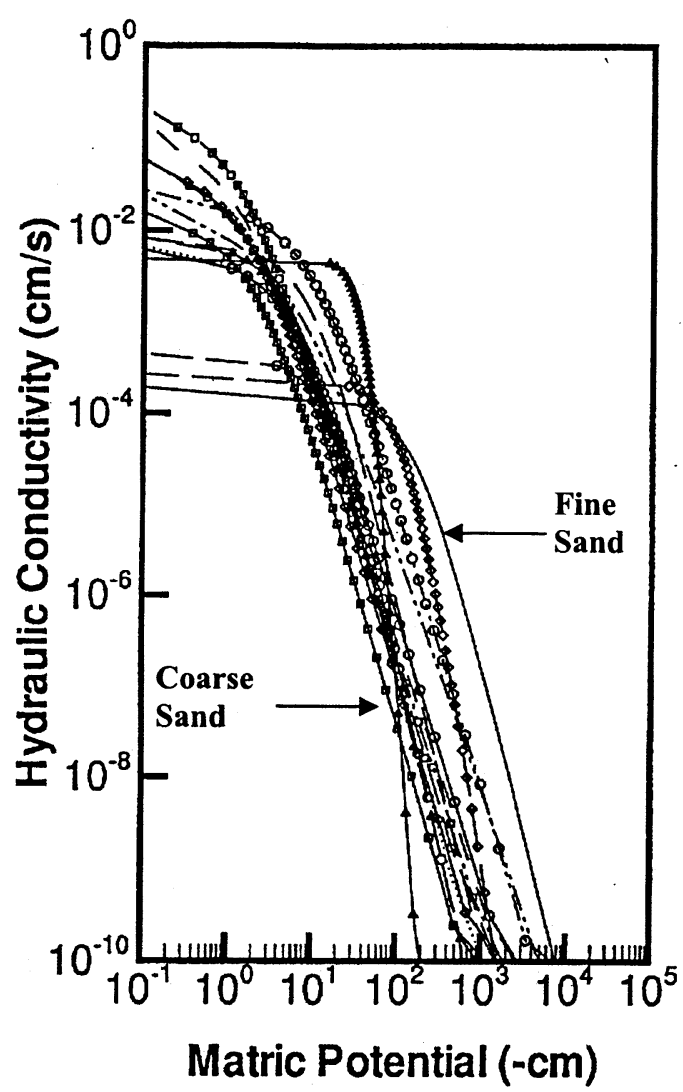

Figure 2. Fitted van Genuchten-Mualem moisture retention and unsaturated conductivity curves for 15 samples. 

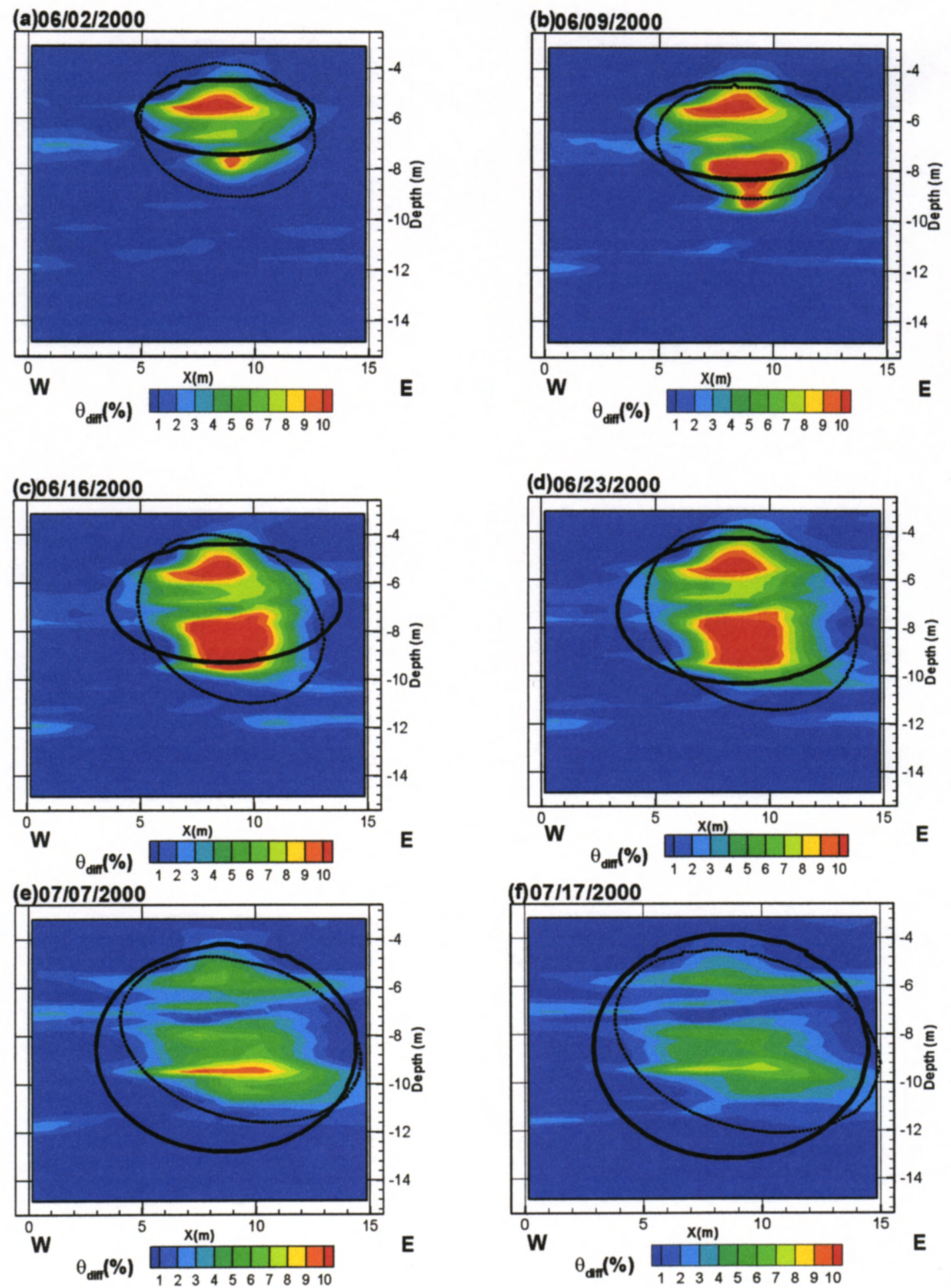

Figure 3. Snapshots of the observed moisture plumes and the ellipsoids (dotted, based on the observed plume and solid, the simulated plume). 

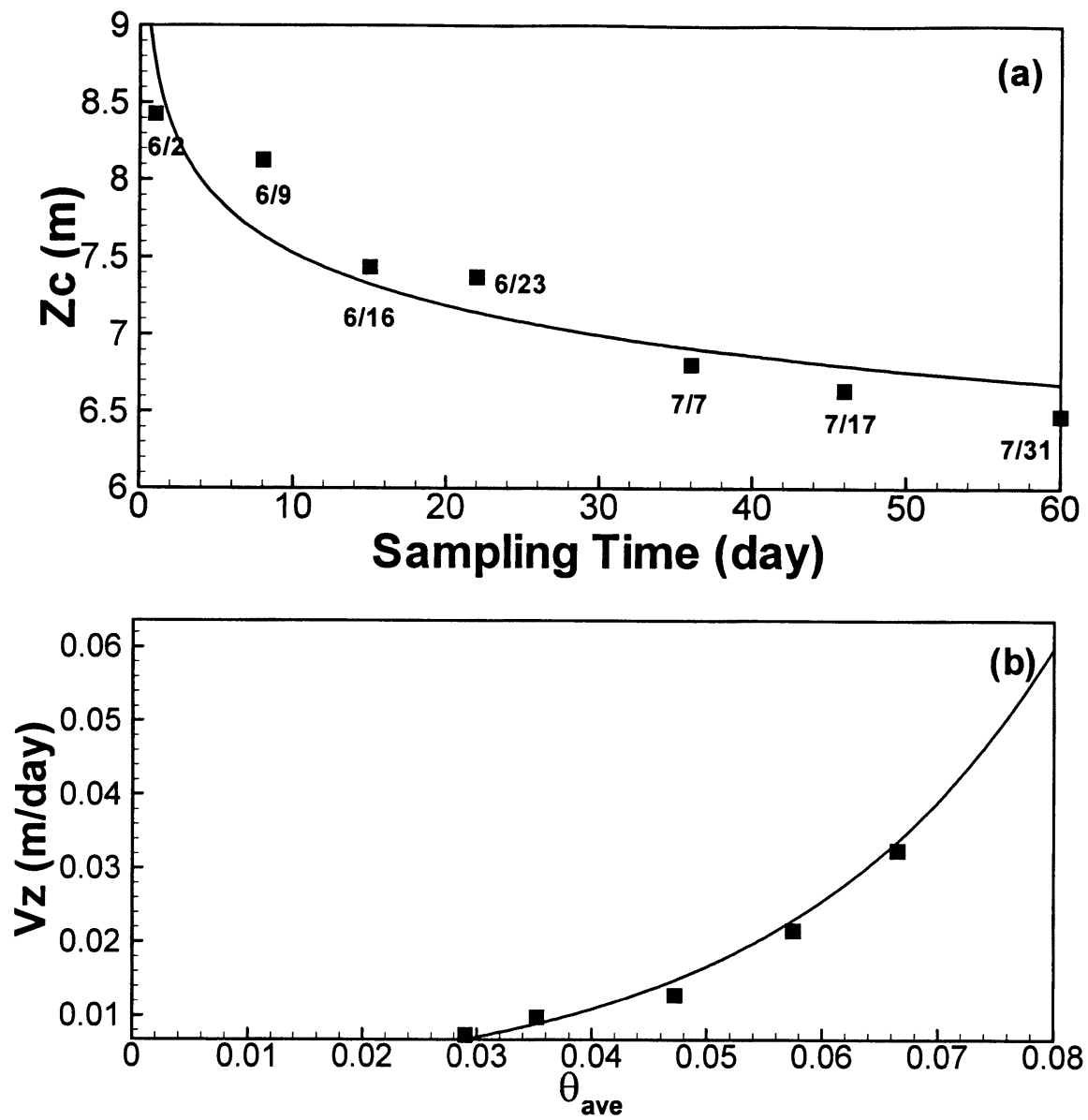

Figure 4. a) Mass centers in the $z$ direction on different days and the best-fit function; b) $V_{z}$ as a function of $\theta_{\text {ave }}$ and the best-fit function for the estimated unsaturated hydraulic conductivity in the $\mathrm{z}$ direction. 

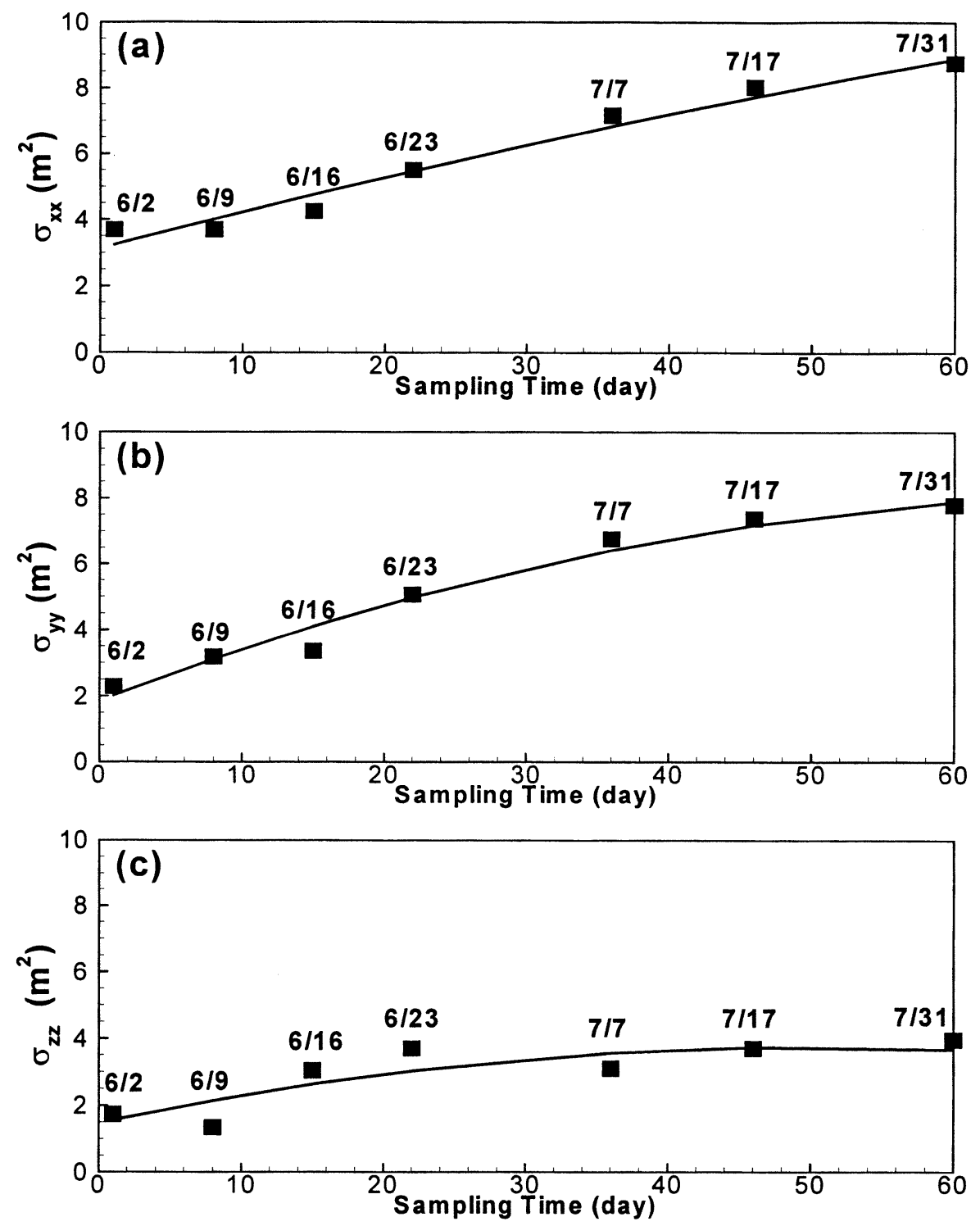

Figure 5. The spatial variances of the observed moisture plume on different days and the best-fit functions describing evolution of the variances as a function of time in the $\mathrm{x}, \mathrm{y}$, and $\mathrm{z}$ directions (a, b, and c, respectively). 


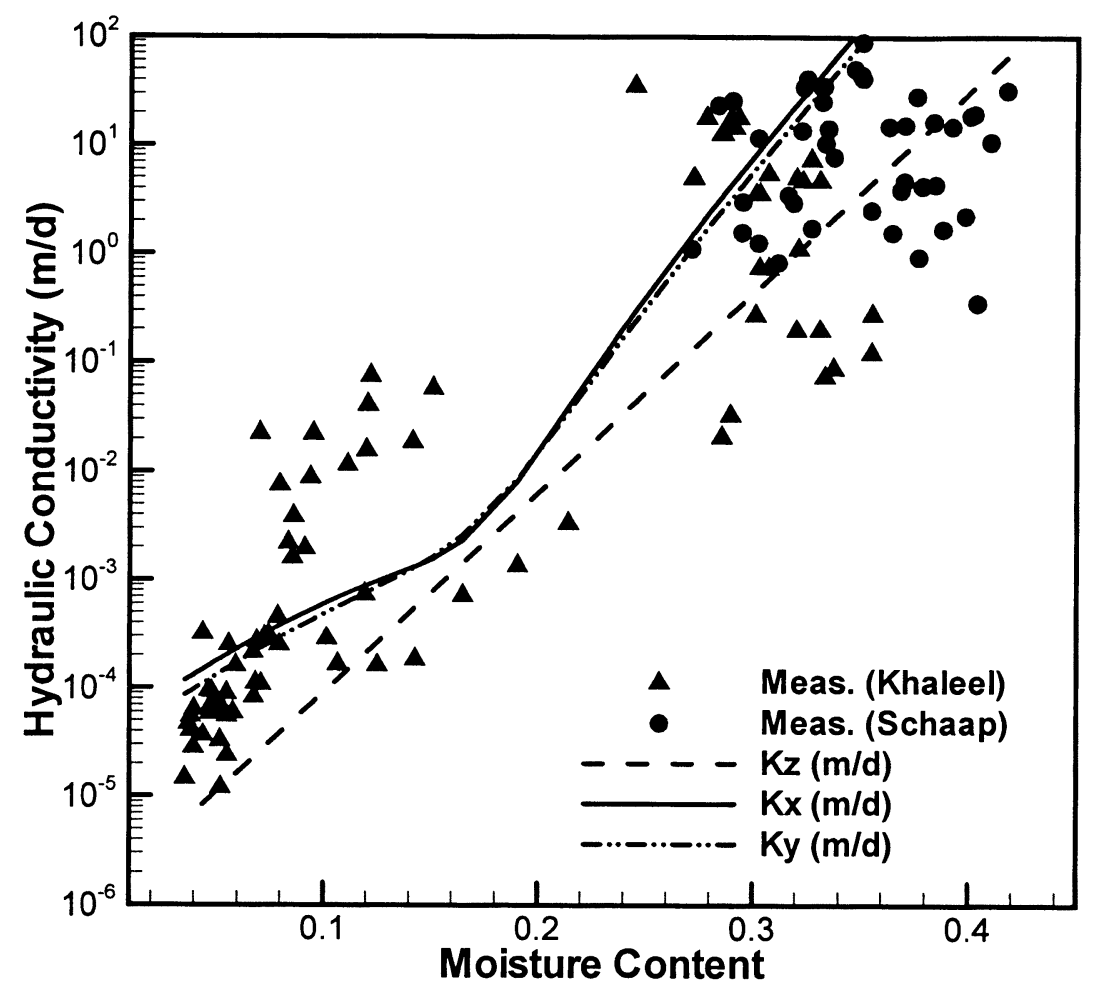

Figure 6. Estimated hydraulic conductivity versus moisture content using our new approach.

Measured unsaturated (Khaleel et al., 1995) and saturated hydraulic conductivity (Schaap, et al., 2003) values from core samples are presented as triangles and circles, respectively. 


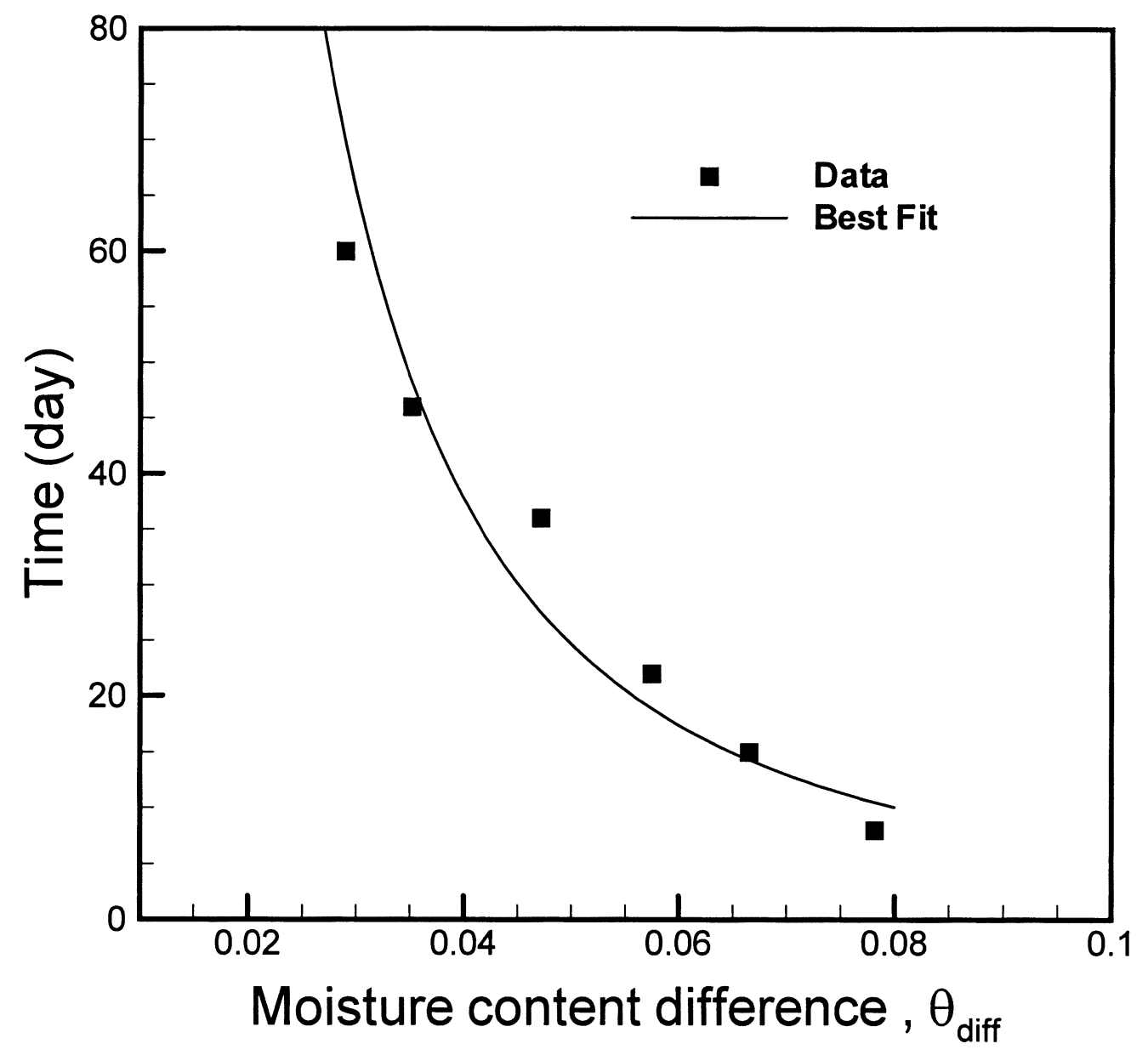

Figure 7. Relation between $t$ and $\theta_{\text {diff }}$. 


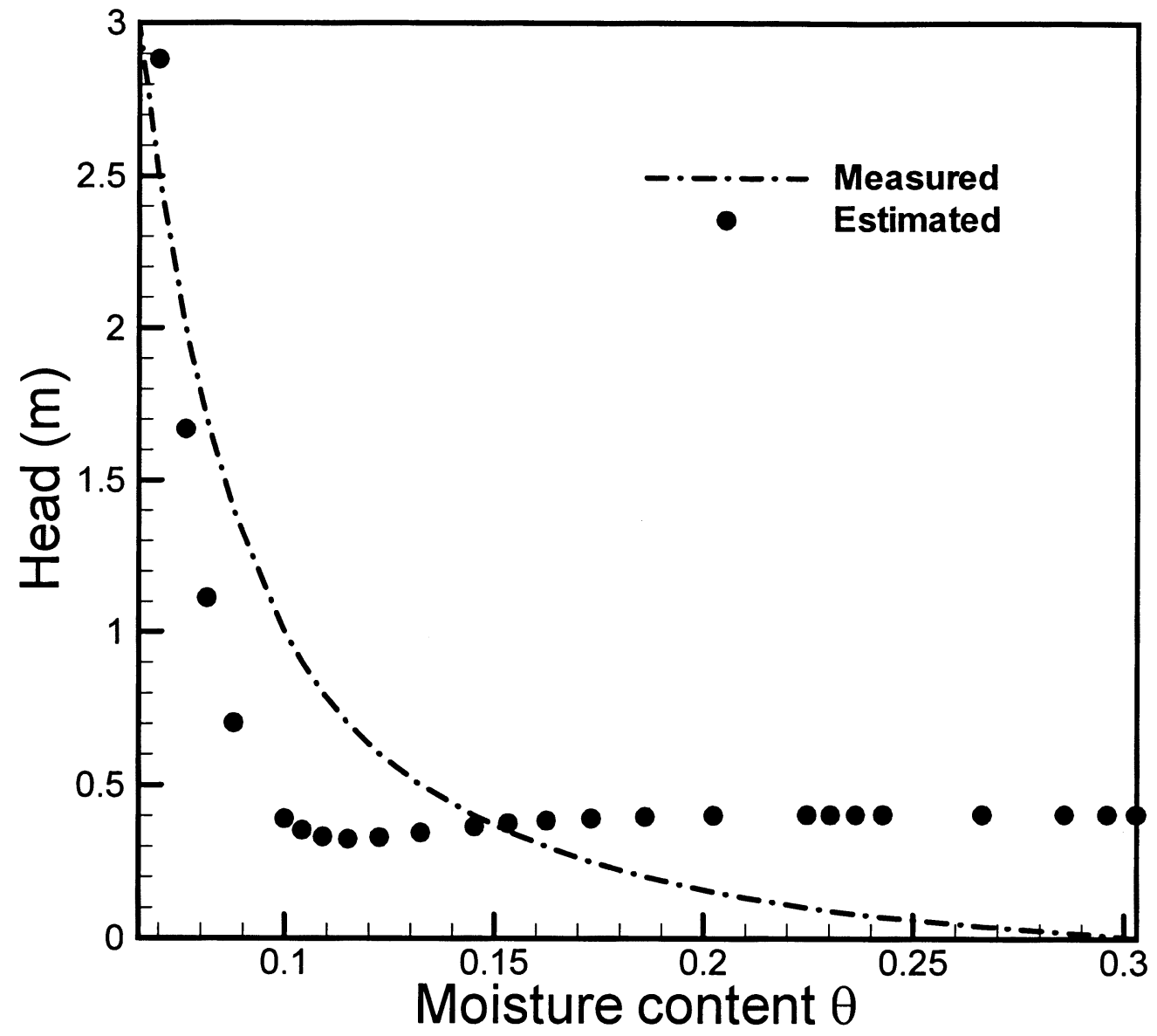

Figure 8. Plot of $h$ versus $\theta$ estimated from the new approach and those from an average of 15 samples. 

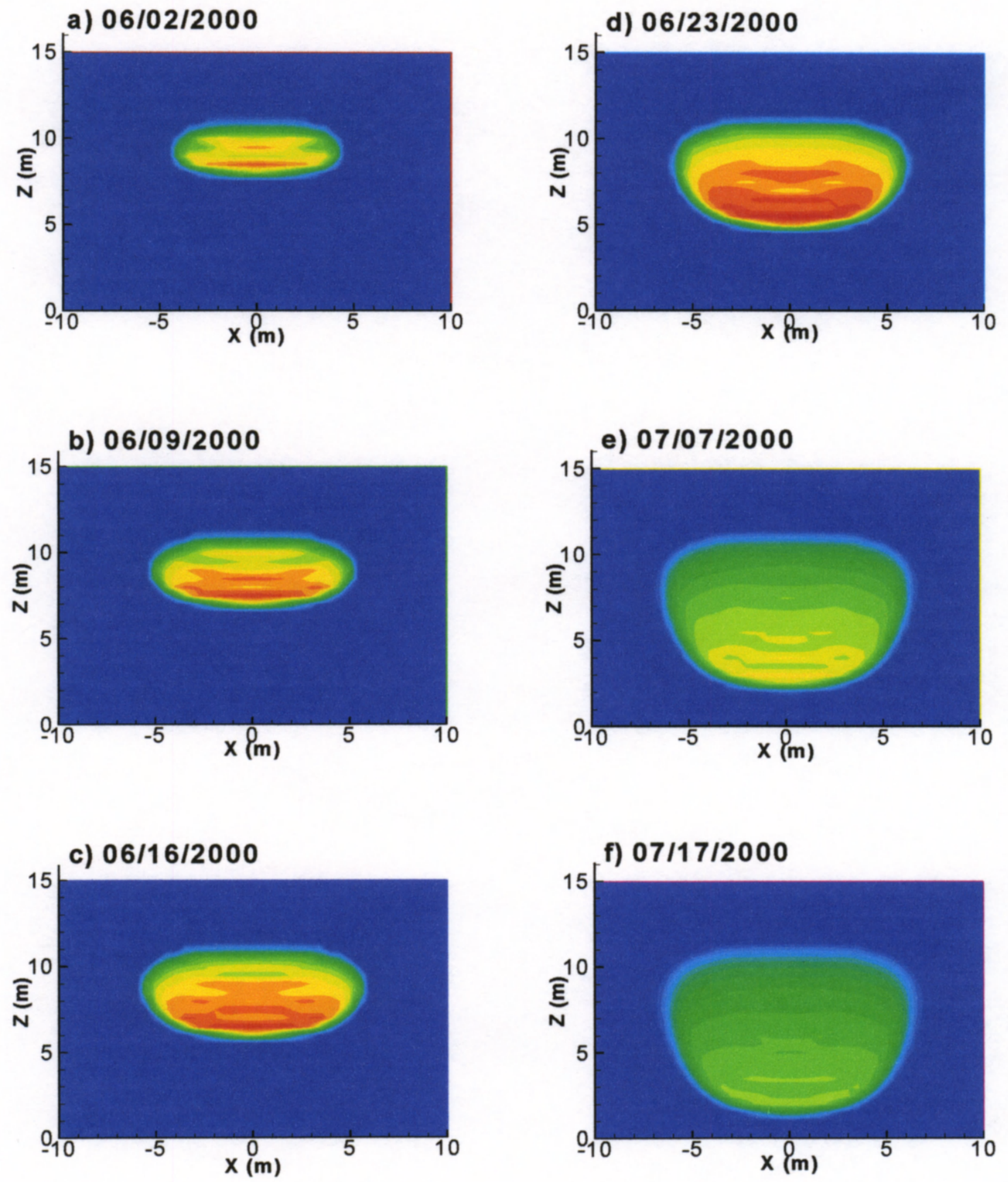

Figure 9. Simulated moisture plume using the effective hydraulic properties based on our new method. 


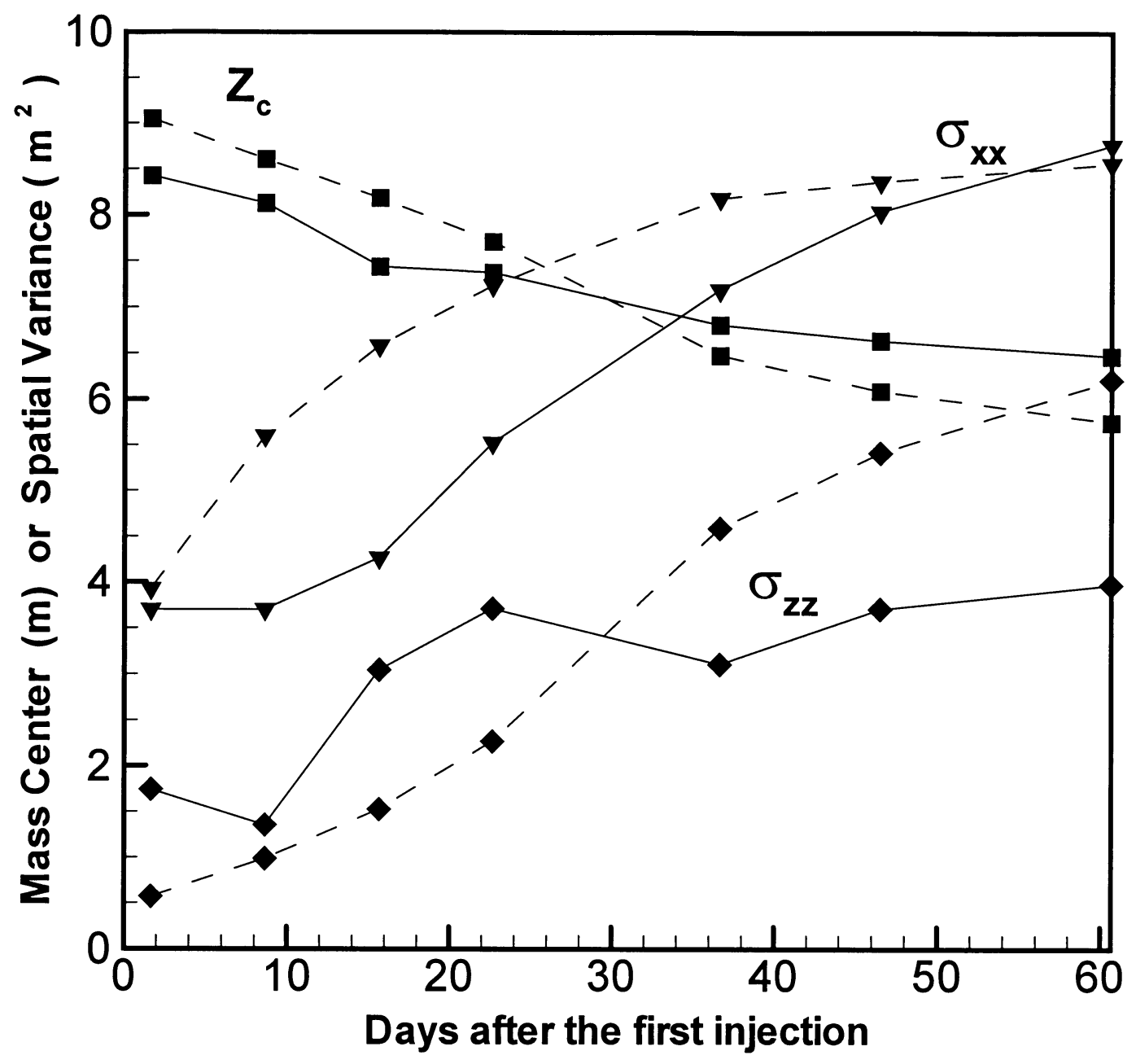

Figure 10. Comparison of mass centers and spatial variances of the simulated (dashed) and observed (solid) moisture plumes. 


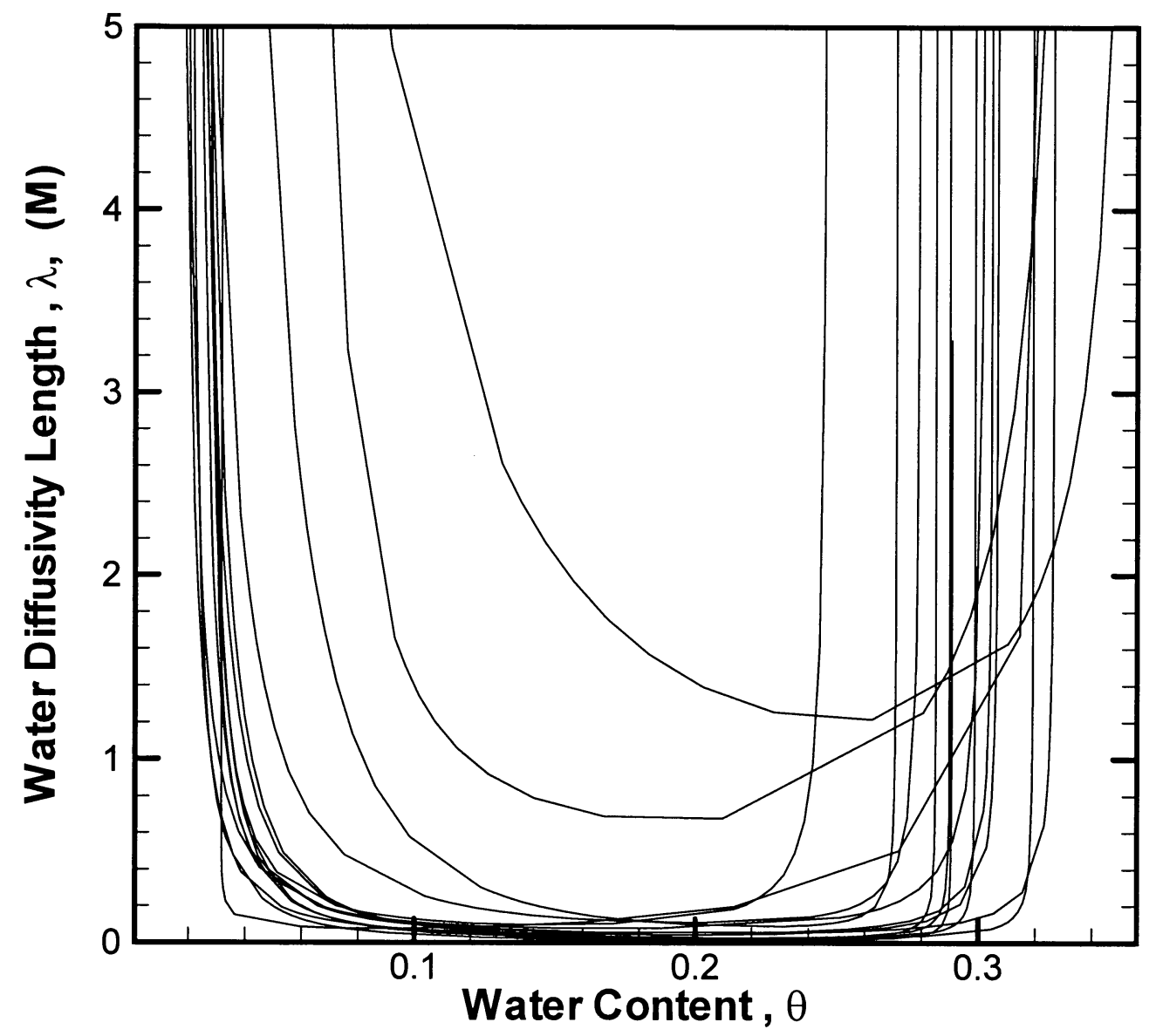

Figure 11. Water diffusivity length and moisture content relations for the 15 soil samples. 